\title{
Fanconi syndrome related to zoledronic acid
}

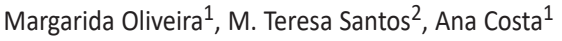 \\ ${ }^{1}$ Department of Medicine, Hospital Pedro Hispano, Matosinhos Local Health Unit, Matosinhos, Portugal \\ 2 Nephrology Unit, Hospital Pedro Hispano, Matosinhos Local Health Unit, Matosinhos, Portugal
}

\begin{abstract}
:
Fanconi syndrome is characterized by proximal tubular dysfunction, with inability to reabsorb bicarbonate causing type 2 renal tubular acidosis associated with aminoaciduria, normoglycemic glycosuria, hypophosphatemia with phosphaturia and proteinuria.

A 68-year-old man with a history of type 2 diabetes mellitus, high-grade papillary carcinoma of the bladder, submitted to transurethral resection in 2011 and 2013 had prostatic adenocarcinoma diagnosed in 2015 with bone metastasis under zoledronic acid. The patient was referred to the emergency department because of anemia "de novo" and acute on chronic kidney injury. Urinary sediment showed nonnephrotic proteinuria and renal ultrasound was normal. He had hyperchloremic metabolic acidosis with hypokalemia, hypophosphatemia, hypocalcemia, hypouricemia, without glycosuria. No evidence of hemolysis nor abnormalities in serum and urinary immunoelectrophoresis, endoscopic studies, myelogram, or bone marrow biopsy. Renal biopsy was compatible with acute tubular necrosis superimposed on diabetic and hypertensive nephropathy. Zoledronic acid was suspended and we observed slow improvement of renal function and resolution of metabolic acidosis and ionic disorders.

Zoledronic acid is nephrotoxic and may induce tubular dysfunction, which can cause Fanconi syndrome. Since 2012, there have been cases relating the administration of zoledronic acid for bone metastasis or hypercalcemia associated with malignant neoplasia with Fanconi syndrome. Although it is a rare association, it is a potentially fatal complication and renal function monitoring is essential.
\end{abstract}

Key words: Fanconi Syndrome, Nephrotoxicity, Renal tubular acidosis, Zoledronic Acid

\section{INTRODUCTION}

Metabolic acidosis can result either from the gain of endogenous or exogenous acids or from the loss of bases through a gastrointestinal or genitourinary route. Renal tubular acidosis (RTA) results from the inability of the kidney to excrete acids or to reabsorb bases, resulting in hyperchloremic metabolic acidosis (with normal anion gap) ${ }^{1}$. Type 2 (or proximal) renal tubular acidosis results from a bicarbonate resorption defect that may occur alone or more often occurs in the context of a global proximal renal tubule dysfunction known as Fanconi syndrome $(\mathrm{FS})^{1,2}$.

Most of the plasma is filtered in the glomerulus and reabsorbed in the proximal contoured tubule. Solutes such as amino acids, phosphate and glucose enter the cell through the apical membrane of the proximal tubule through a $\mathrm{Na}^{+}$co-transporter. Sodium transport is potentiated by the $\mathrm{Na}^{+} / \mathrm{K}^{+}$ATPase in the basal membrane of the cell that leads to $\mathrm{Na}^{+}$absorption and its intracellular decrease ${ }^{3}$.

Proximal tubular dysfunction leads to urinary loss of substances that are predominantly or exclusively reabsorbed in this segment, namely amino acids, low molecular weight proteins, phosphate, bicarbonate, glucose and uric acid ${ }^{4}$. This leads to the features of $\mathrm{FS}^{4}$. The proximal tubule also reabsorbs a significant portion of sodium, potassium, chloride, magnesium and calcium ${ }^{4}$. Thus, FS is characterized not only by hyperchloremic metabolic acidosis but also hypokalemia, aminoaciduria, proteinuria (mainly low molecular weight proteins), phosphaturia, glycosuria. Other manifestations are hypouricemia, hyponatraemia, hypercalciuria and polyuria ${ }^{5}$. Some patients appear to have only some of the characteristics of Fanconi syndrome ${ }^{4}$, such as isolated tubular proteinuria. FS may or may not be associated with glomerular disease, and there may be severe tubular dysfunction without worsening of the glomerular filtration rate ${ }^{4}$.

The causes of FS range from hereditary diseases to acquired causes ${ }^{5}$. Hereditary diseases include cystinosis, mitochondrial cytopathy, tyrosinemia, fructose intolerance, galactosemia, Dent's disease, Lowe's syndrome and Wilson's disease ${ }^{4,5}$. Among the acquired causes, the most common is pharmacological toxicity. Other causes of acquired FS are Sjögren's syndrome, amyloidosis, multiple myeloma, monoclonal gammopathy, and light chain deposition disease and obstructive uropathy ${ }^{6}$.

\section{CASE REPORT}

The authors present the case of a 68-year-old man with type 2 diabetes mellitus diagnosed in 2003 under oral antidiabetic drugs, $\mathrm{HbA} 1 \mathrm{C} 6.6 \%$ and microalbuminuria (110mg/g), no diabetic retinopathy, dyslipidemia, hypertension, chronic renal disease (which has not been previously studied), with a glomerular filtration rate (GFR) of $58 \mathrm{ml} /$ $\mathrm{min} / 1.73 \mathrm{~m}^{2}$ estimated by the CKD-EPI formula (stage 3a) with albuminuria $110 \mathrm{mg} / \mathrm{g}$ (A2) in 2015. He had documented normal renal function before 2010 and no history of deafness. The patient had high grade papillary carcinoma of the bladder, submitted to transurethral 


\section{Table I}

Summary of laboratory analysis

\begin{tabular}{|c|c|c|}
\hline Blood analysis & & Reference value \\
\hline Hemoglobin (g/dl) & 6.9 & $13-18$ \\
\hline Hematocrit (\%) & 19.9 & $40-52$ \\
\hline $\mathrm{Cr}(\mathrm{mg} / \mathrm{dL})$ & 2.7 & $0.7-1.3$ \\
\hline Ureia (mg/dL) & 97 & $18-55$ \\
\hline $\mathrm{Na}^{+}(\mathrm{mEq} / \mathrm{L})$ & 138 & $136-145$ \\
\hline $\mathrm{K}^{+}(\mathrm{mEq} / \mathrm{L})$ & 3.3 & $3.4-5.1$ \\
\hline $\mathrm{Cl}^{-}(\mathrm{mEq} / \mathrm{L})$ & 117 & $98-107$ \\
\hline $\mathrm{PO}_{4}^{3-}(\mathrm{mg} / \mathrm{dL})$ & 2.4 & $2.4-4.7$ \\
\hline $\mathrm{Mg}^{2+}(\mathrm{mg} / \mathrm{dL})$ & 1.35 & $1.6-2.6$ \\
\hline $\mathrm{Ca}^{2+}(\mathrm{mmol} / \mathrm{L})$ & 1.07 & $1.15-1.35$ \\
\hline Uric acid (mg/dL) & 3.2 & $3.5-7.2$ \\
\hline Total proteins (g/dL) & 6.2 & $6.4-8.3$ \\
\hline $\mathrm{pH}$ & 7.32 & $7.35-7.45$ \\
\hline $\mathrm{pCO}_{2}(\mathrm{mmHg})$ & 20 & $35-40$ \\
\hline $\mathrm{HCO}_{3}^{-}(\mathrm{mmol} / \mathrm{L})$ & 10.3 & $18-23$ \\
\hline
\end{tabular}

resection in 2011 and 2013 and prostate adenocarcinoma (Gleason $4+3$ ) with bone metastasis, diagnosed in February 2015 and since then under triptorelin, cyproterone acetate and zolendronic acid $4 \mathrm{mg}$ ( 1 intravenous administration for 15 minutes, every 4 weeks).

On April 2016, he was referred to the Emergency Department by his primary health care physician due to asthenia, anorexia and anemia "de novo" (last normal value in 2015). On objective examination he had mucocutaneous pallor, without any other abnormal findings. The analytical study (Table I) showed normocytic and normochromic anemia (hemoglobin $6.9 \mathrm{~g} / \mathrm{dL}$ ) and acute on chronic kidney injury AKIN 2 (plasma creatinine $2.7 \mathrm{mg} / \mathrm{dL}$, for a previous value of $1.3 \mathrm{mg} / \mathrm{dL}$ in 2015 ). Urinalysis with proteinuria $(110 \mathrm{mg} / \mathrm{dL})$, without any other abnormal findings. Renal ultrasound showed no abnormalities in renal or urinary tract structure. Nevertheless, he had compensated hyperchloremic metabolic acidosis (pH 7.32, $\left.\mathrm{pCO}_{2} 20 \mathrm{mmHg}, \mathrm{HCO}_{3}{ }^{-} 10.3 \mathrm{mmol} / \mathrm{L}, \mathrm{Cl}^{-} 117 \mathrm{mmol} / \mathrm{L}\right)$, urine $\mathrm{pH} 6.5$ and hypokalemia $(3.3 \mathrm{mmol} / \mathrm{L})$, hypocalcemia $(1.07 \mathrm{mg} / \mathrm{L})$, hypomagnesemia $(1.35 \mathrm{mg} / \mathrm{dL}$ ) and hypophosphatemia $(2.4 \mathrm{mg} / \mathrm{dL})$.

The patient had no history of diarrhea, so the main hypothesis was that of renal tubular acidosis justifying hyperchloremic metabolic acidosis and hypokalemia. Urine analysis showed an increased bicarbonate excretion fraction of $16.4 \%$ (for a normal range $<15 \%$ ), supporting the diagnosis of renal tubular acidosis type 2 .

Urinary amino acids analysis (Table II) showed an increase of 5 of 17 measured amino acids, non-selective proteinuria, hypophosphatemia with phosphaturia, hypocalcemia with calciuria, hypokalemia with kaliuria and hypouricemia with uricosuria, without glycosuria. Thus, we concluded we were facing a proximal tubular dysfunction as in FS.

Considering the patient had acute on chronic kidney injury and anemia, it was mandatory to suspect $f$ multiple myeloma, light-chain disease or amyloidosis. To complete the study, we found a low reticulocyte index (0.64\%), with a slight deficit of vitamin B12 (183pg/mL),

\section{Table II}

Urinary amino acids analysis

\begin{tabular}{|c|c|c|}
\hline Amino acid & & Reference value (mg/L/24h) \\
\hline Alanine & 47 & $8-48$ \\
\hline Arginine & 1 & $<50$ \\
\hline Aspartic acid & 1 & $<27$ \\
\hline Cystine & 53,9 & $<38$ \\
\hline Phenylalanine & 24 & $<17$ \\
\hline Glutamic acid & 49,2 & $<34$ \\
\hline Glycine & 45 & $54-294$ \\
\hline Histidine & 51 & $73-441$ \\
\hline Isoleucine & 2,9 & $2-24$ \\
\hline Lysine & 13 & $3-153$ \\
\hline Leucine & 14 & $3-70$ \\
\hline Methionine & 1,4 & $<9$ \\
\hline Proline & 28,3 & Vestigial \\
\hline Serine & 8 & $14-126$ \\
\hline Threonine & 22 & $15-53$ \\
\hline Tyrosine & 13 & $12-55$ \\
\hline Valine & 34 & $2-12$ \\
\hline
\end{tabular}

without any change in iron kinetics or folate deficiency. No changes were also found in serum and urinary immunoelectrophoresis. Myelogram showed hypocellularity with $1 \%$ plasma cells, with no qualitative abnormalities, and bone marrow biopsy showed no qualitative nor quantitative deviations nor any sign of malignancy or amyloid deposits. Upper and lower digestive endoscopy were normal with no amyloid deposits in rectal fat biopsy. Hence, there was no evidence of any of the postulated diagnostic hypotheses. We extended the study to autoimmune paraneoplastic causes, but ANA, anti-dsDNA, anti-SSA and anti-SSB came out negative. We documented vitamin $D$ deficiency $(9 \mathrm{U} / \mathrm{L})$, but it was not possible to establish it as a cause or an effect of the disease. Renal biopsy (Fig. 1-4) was performed on 04/29/2016, showing acute tubular necrosis superimposed on diabetic nephropathy without significant expression at glomerular level and vascular lesions suggestive of hypertensive nephropathy.

In view of these findings, the patient's previous susceptibility to renal damage due to diabetic and hypertensive nephropathy and having excluded other causes, we considered the diagnosis of zoledronic acid nephrotoxicity, and, once the zoledronic acid was suspended, the patient showed a progressive, but slow, improvement in tubular function. In 2 months, we observed the complete resolution of metabolic acidosis and ionic disorders with no need of supplementation, but the improvement in glomerular filtration rate was much slower. On January 2018, the patient had for the first time a plasma creatinine of $1.5 \mathrm{mg} / \mathrm{dL}$, with an estimated GFR of $45 \mathrm{ml} / \mathrm{min} / 1.73 \mathrm{~m}^{2}$.

\section{DISCUSSION}

Zoledronic acid is a nitrogenated bisphosphonate with a half-life of $150-200$ days and a low binding rate to plasma proteins $(56 \%)^{7,8}$. It inhibits bone resorption in patients with post-menopausal 


\section{Figure 1-4}

Renal biopsy showing toxic acute tubular necrosis (proximal tubules exhibit severe degenerative changes including luminal ectasia, cytoplasmatic simplification and hypereosinophilia, irregular luminal contours, extensive loss of brush border, proeminent nucleoli). (Hematoxylin and eosin)
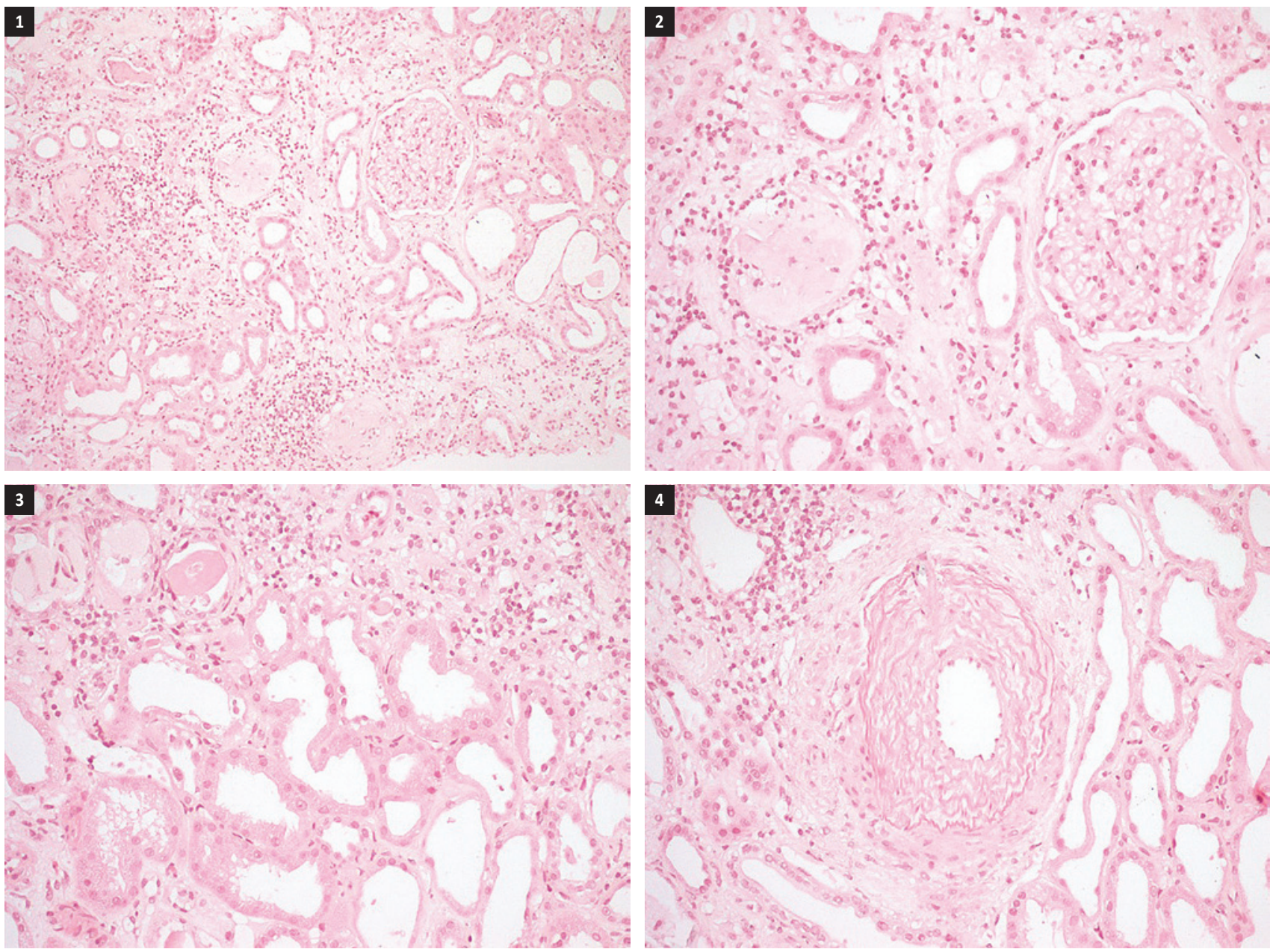

osteoporosis, hypercalcemia due to malignancy, Paget's disease and bone metastases associated with solid tumors and multiple myelo$\mathrm{ma}^{5,8,9}$. Nephrotoxicity is described for all the bisphosphonate class, but it is more frequent with higher doses and potency (namely pamidronate and zoledronic acid) and with intravenously administration $^{8}$. When administered intravenously, biphosphonates are not metabolized, and are excreted unaltered by the kidneys ${ }^{7,10}$ through glomerular filtration ${ }^{8}$ and a small percentage is actively secreted at the tubular level. As a result, when the renal function is impaired, bisphosphonate excretion is reduced which can lead to excessive serum and bone levels resulting in toxicity ${ }^{8}$. Bisphosphonate nephrotoxicity is both dose-dependent and infusion time-dependent ${ }^{8}$. Patterns in renal biopsies include acute tubular necrosis, segmental and focal glomerulosclerosis and a case of acute interstitial nephritis was described $^{8-10}$.

Acute tubular necrosis, the main pattern of injury seen in patients with zoledronic acid nephrotoxicity, is characterized by toxicity directed at tubular epithelium ${ }^{8}$. This toxicity can be expressed in a number of ways, namely by reducing the permeability of the apical membrane, by failure of endocytosis and apical transporters, by lysosomal dysfunction, by glutathione depletion, by mitochondrial toxicity and consequently a decrease in ATP production or by direct inhibition of ATPase $\mathrm{Na}^{+} / \mathrm{K}^{+4,8}$. It is believed that bisphosphonate nephrotoxicity results from direct damage to the tubular cells by inducing apoptosis through the mevalonate pathway, in a similar way to its apoptotic effect on osteoclasts ${ }^{8,9}$.

The estimated incidence of acute tubular necrosis associated to zoledronic acid is $2-10 \%{ }^{9}$. In a 2003 study published in the NEJM ${ }^{10}, 72$ cases were identified by the FDA between 2001 and 2003 of zoledronic acid-associated renal dysfunction, with 6 of these referring to patients with prostate cancer ${ }^{10}$. The mean time from onset of treatment to the detection of renal failure was 56 days (1-242 days), with a mean of 2.4 administrations. In this series ${ }^{10}, 48$ patients required hospitalization, 27 patients needed dialysis and 18 died. The majority of patients had 
a baseline creatinine level of $1.4-3 \mathrm{mg} / \mathrm{dL}$, with a mean exacerbation up to $6.5 \mathrm{mg} / \mathrm{dL}$ during treatment with zoledronic acid and recovery to $2.7 \mathrm{mg} / \mathrm{dL}$ after discontinuation of the drug. In this case, the time from onset of administration to the onset of the first symptoms was about 365 days, with a cumulative dose of $40 \mathrm{mg}$, with a previous creatinine of $1.3 \mathrm{mg} / \mathrm{dL}$ and a maximum of $3.3 \mathrm{mg} / \mathrm{dL}$ and recovery to $1.5 \mathrm{mg} / \mathrm{dL}$ after discontinuation of the drug. In all the reported cases, the treatment is the suspension of the nephrotoxic drug and the ionic supplementation as required, as in the present case.

While acute kidney injury is infrequent, FS is quite rare. FS seems to be the result of an overall dysfunction of solute transport at the proximal tubule level ${ }^{4}$. It is believed that the incidence of renal tubular toxicity is underestimated by the absence of systematic studies to monitor tubular dysfunction (and not only a decrease in GFR) and underreporting of cases $^{4}$. Although the renal tubules have a great regenerative capacity, this can take months and it may not always be complete, leading to chronic disfunction of renal tubules even after the withdrawal of the drug ${ }^{4}$. The first reported case of FS related to zoledronic acid was in 2012. Since then, there have been other cases in which the administration of zoledronic acid was associated with serum and urinary electrolyte abnormalities compatible with FS, improving after drug withdrawal.

This case brings together several of Naranjo's criteria for adverse drug reaction, namely: the occurrence of the adverse event after exposure to the drug, improvement after discontinuation of the drug, lack of a better diagnostic explanation and the existence of other scientific reports documenting this association ${ }^{11}$.

The authors note that zoledronic acid has been shown to be of benefit in patients with bone metastasis, with a well-established safety profile when used properly ${ }^{10}$. Its nephrotoxic potential does not contraindicate its use, especially in cases where the benefits outweigh the risks ${ }^{4}$. The Food and Drug Administration recommends monitoring serum creatinine prior to each treatment and adjusting doses in patients with chronic kidney disease and treatment should be withheld when there is an increase of $0.5 \mathrm{mg} / \mathrm{dL}$ in patients with previous normal renal function or $1 \mathrm{mg} / \mathrm{dL}$ in patients with previous abnormal renal function ${ }^{8}$. Nevertheless, the European Commission permits renal function to be monitored at the discretion of the physician ${ }^{8}$. It is also recommended to respect infusion time ${ }^{8}$. We should note that despite the patient's GFR being $58 \mathrm{ml} / \mathrm{min} / 1.73 \mathrm{~m}^{2}$ in 2015 , zoledronic acid was administered in full dose and no monitoring of creatinine clearance was made before each dose.
We emphasize it is essential to monitor renal function and ensure adequate hydration, with special attention being paid to patients with diabetes mellitus and hypertension, as was the case with this patient (although hypertension was not previously diagnosed or treated).

Since there is not always a decrease in the rate of glomerular filtration associated with $\mathrm{FS}$, monitoring of proximal tubular function during therapy with zoledronic acid is also recommended ${ }^{4,5}$.

\section{Acknowledgments}

To Professor Rui Henrique for kindly providing the renal biopsy images.

Disclosure of potential conflicts of interest: none declared

\section{References}

1. Soleimani M, Rastegar A. Pathophysiology of renal tubular acidosis: Core Curriculum 2016. Am J Kidney Dis. 2016:68(3):488-498

2. Perrone D, Afridi F, King-Morris K, Komarla A, Kar P. Proximal renal tubular acisosis (Fanconi Syndrome) induced by apremilast: A case report. Am J Kidney Dis. 2017;70(5):729-731

3. Possible Fanconi Syndrome associated with zoledronic acid. Available at: http://www.hsa.gov.sg/ content/hsa/en/Helth_Products_Regulation/Safety_Information_and_Product_Recalls/Product_Safety_Alerts/2015/possible-fanconisyndromeassociatedwithzoledronicacid.html. Accessed May 3, 2016

4. Hall AM, Bass P, Unwin RJ. Drug-induced renal Fanconi Syndrome. QJM. 2014;107(4):261-269

5. Yoshinami T, Yagi T, Sakai D, Sugimoto N, Imamura F. A case of acquired Fanconi Syndrome induced by zoledronic acid. Intern Med. 2011;50(9):1075-1079

6. Yaxley J, Pirrone C. Review of the diagnostic evaluation of renal tubular acidosis. Ochsner J. 2016;16(4):525-530

7. Gutiérrez Sánchez MJ, Petkov Stoyano V, Pedraza Cezón L, Martín Navarro JA, Insuficiencia renal por nefropatia tubulointersticial com tubulopatía proximal tipo Fanconi tras tratamento com ácido zoledrónico. Nefrología. 2017;37:660-661

8. Perazella MA, Markowitz GS. Bisphosphonate nephrotoxicity. Kidney Int. 2008;74(11):1385-1393

9. Torimoto K, Okada Y, Arao T, Mori H, Tanaka Y. A case of zoledronate-induced tubulointerstitial nephritis with Fanconi Syndrome. Endocr J. 2012;59(12):1051-1056

10. Chang JT, Green L, Beitz J. Renal failure with the use of zoledronic acid. N Engl J Med. 2003;349(17):1676-1679

11. Naranjo CA et al. A method for estimating the probability of adverse drug reactions. Clin Pharmacol Ther. 1981;30(2):239--245

\section{Correspondence to:}

Margarida Oliveira, MD

Department of Medicine, Hospital Pedro Hispano

Rua Dr. Eduardo Torres, 4464-513 Senhora da Hora, Matosinhos, Portugal E-mail: med6084@ulsm.min-saude.pt 